\title{
Generation of human liver organoids from pluripotent stem cell-derived hepatic endoderms
}

\author{
Kasem Kulkeaw ${ }^{\text {Corresp., }}{ }^{1}$, Alisa Tubsuwan ${ }^{2}$, Nongnat Tongkrajang ${ }^{1}$, Narisara Whangviboonkij ${ }^{1}$ \\ ${ }^{1}$ Department of Parasitology, Faculty of Medicine Siriraj Hospital, Mahidol University, Bangkok, Thailand, Thailand \\ 2 Stem Cell Research Group, Institute of Molecular Biosciences, Mahidol University, Nakhon Pathom, Thailand \\ Corresponding Author: Kasem Kulkeaw \\ Email address: kasem.kuk@mahidol.edu
}

Background. The use of a personalized liver organoid derived from human-induced pluripotent stem cells (HuiPSCs) is advancing the use of in vitro disease models for the design of specific, effective therapies for individuals. Collecting patient peripheral blood cells for HuiPSC generation is preferable because it is less invasive; however, the capability of blood cell-derived HuiPSCs for hepatic differentiation and liver organoid formation remains uncertain. Moreover, the currently available methods for liver organoid formation require a multistep process of cell differentiation or a combination of hepatic endodermal, endothelial and mesenchymal cells, which is a major hurdle for the application of personalized liver organoids in high-throughput testing of drug toxicity and safety. To demonstrate the capability of blood cell-derived HuiPSCs for liver organoid formation without support from endothelial and mesenchymal cells.

Methods. The peripheral blood-derived HuiPSCs first differentiated into hepatic endoderm (HE) in twodimensional (2D) culture on Matrigel-coated plates under hypoxia for 10 days. The HE was then collected and cultured in 3D culture using 50\% Matrigel under ambient oxygen. The maturation of hepatocytes was further induced by adding hepatocyte growth medium containing HGF and oncostatin M on top of the 3D culture and incubating the culture for an additional 12-17 days. The function of the liver organoids was assessed using expression analysis of hepatocyte-specific gene and proteins. Albumin (ALB) synthesis, glycogen and lipid storage, and metabolism of indocyanine were evaluated. The spatial distribution of albumin was examined using immunofluorescence and confocal microscopy.

Results. CD34+ hematopoietic cell-derived HuiPSCs were capable of differentiating into definitive endoderm expressing SOX17 and FOXA2, hepatic endoderm expressing FOXA2, hepatoblasts expressing AFP and hepatocytes expressing ALB. On day 25 of the 2D culture, cells expressed SOX17, FOXA2, AFP and $A L B$, indicating the presence of cellular heterogeneity. In contrast, the hepatic endoderm spontaneously formed a spherical, hollow structure in a 3D culture of 50\% Matrigel, whereas hepatoblasts and hepatocytes could not form. Microscopic observation showed a single layer of polygonal-shaped cells arranged in a 3D structure. The hepatic endoderm-derived organoid synthesis ALB at a higher level than the 2D culture but did not express definitive endoderm-specific SOX17, indicating the greater maturity of the hepatocytes in the liver organoids. Confocal microscopic images and quantitative ELISA confirmed albumin synthesis in the cytoplasm of the liver organoid and its secretion. Overall, 3D culture of the hepatic endoderm is a relatively fast, simple, and less laborious way to generate liver organoids from HuiPSCs that is more physiologically relevant than 2D culture. 
1 Generation of Human Liver Organoids from Pluripotent Stem Cell-Derived Hepatic

2 Endoderms

3

4 Kasem Kulkeaw $^{1}$, Alisa Tubsuwan ${ }^{2}$, and Nongnat Tongkrajang ${ }^{1}$ and Narisara Whangviboonkij 5

$6{ }^{1}$ Department of Parasitology, Faculty of Medicine Siriraj Hospital, Mahidol University,

7 Bangkok, Thailand

$8{ }^{2}$ Stem Cell Research Group, Institute of Molecular Biosciences, Mahidol University, Nakhon

9 Pathom, Thailand

10

12 Corresponding Author:

13 Kasem Kulkeaw ${ }^{1}$

142 Wanglang Road, Bangkok-Noi, Bangkok 10700, Thailand

15 Email address: kasem.kuk@mahido.edu

16

17

18

19

20

21

22

23

24

25

26

27

28

29

30

31

32

33

34

35

36

37

38

39

40

41 


\section{Abstract}

43 Background. The use of a personalized liver organoid derived from human-induced pluripotent

44 stem cells (HuiPSCs) is advancing the use of in vitro disease models for the design of specific, effective therapies for individuals. Collecting patient peripheral blood cells for HuiPSC generation is preferable because it is less invasive; however, the capability of blood cell-derived HuiPSCs for hepatic differentiation and liver organoid formation remains uncertain. Moreover, the currently available methods for liver organoid formation require a multistep process of cell differentiation or a combination of hepatic endodermal, endothelial and mesenchymal cells, which is a major hurdle for the application of personalized liver organoids in high-throughput testing of drug toxicity and safety.bTo demonstrate the capability of blood cell-derived HuiPSCs for liver organoid formation without support from endothelial and mesenchymal cells

Methods. The peripheral blood-derived HuiPSCs first differentiated into hepatic endoderm in two-dimensional (2D) culture on Matrigel-coated plates under hypoxia for 10 days. The HE was then collected and cultured in 3D culture using 50\% Matrigel under ambient oxygen. The maturation of hepatocytes was further induced by adding hepatocyte growth medium containing HGF and oncostatin M on top of the 3D culture and incubating the culture for an additional 1217 days. The function of the liver organoids was assessed using expression analysis of hepatocyte-specific gene and proteins. Albumin (ALB) synthesis, glycogen and lipid storage, and metabolism of indocyanine were evaluated. The spatial distribution of albumin was examined using immunofluorescence and confocal microscopy.

Results. CD34+ hematopoietic cell-derived HuiPSCs were capable of differentiating into definitive endoderm expressing $S O X 17$ and $F O X A 2$, hepatic endoderm expressing $F O X A 2$, hepatoblasts expressing $A F P$ and hepatocytes expressing $A L B$. On day 25 of the $2 \mathrm{D}$ culture, cells expressed $S O X 17, F O X A 2, A F P$ and $A L B$, indicating the presence of cellular heterogeneity. In contrast, the hepatic endoderm spontaneously formed a spherical, hollow structure in a 3D culture of 50\% Matrigel, whereas hepatoblasts and hepatocytes could not form. Microscopic observation showed a single layer of polygonal-shaped cells arranged in a 3D structure. The hepatic endoderm-derived organoid synthesized ALB at a higher level than the 2D culture, indicating the greater maturity of the hepatocytes in the liver organoids. Confocal microscopic images and quantitative ELISA confirmed albumin synthesis in the cytoplasm of the liver organoid and in the culture medium. Overall, 3D culture of the hepatic endoderm is a relatively 
73 fast, simple, and less laborious way to generate liver organoids from HuiPSCs that is more 74 physiologically relevant than $2 \mathrm{D}$ culture.

75

\section{Introduction}

77 Organoids are in vitro three-dimensionally (3D) self-organizing cells capable of imitating the

78

79

80

81

82

83

84

85

86

87

88

89

90 structure and function of tissues or organs originating in the human body. Given their lower complexity and simpler experimental accessibility compared to tissues or organs in the body, organoids are useful for studying organ development (Sato et al. 2009), genetic diseases [Dekkers et al. 2013; Huch et al. 2015], cancers [Boj et al. 2015; Gao et al. 2014; Hubert et al. 2016], and host-pathogen interactions [Bartfeld et al. 2015; Castellanos-Gonzalez et al. 2013; Farin et al. 2014; Zomer-van Ommen et al. 2016] under a physiologically relevant microenvironment. However, to generate an organoid, cells or parts of tissue need to be isolated from an embryo, fetus, or adult organs. Such an invasive procedure has led to the decreased accessibility of adult human samples. Moreover, the limited availability and ethical concerns related to human embryonic and fetal tissues additionally restrict their use. Given advances in stem cell technology, human-induced pluripotent stem cells (HuiPSCs) are an alternative source for generating organoids for studying human brain development (Lancaster et al. 2013), polycystic kidney disease (Xia et al. 2013)] and infectious diseases (Cugola et al. 2016) (Garcez et al. 2016) (Finkbeiner et al. 2012) (Forbester et al. 2015) (Leslie et al. 2015).

Owing to the physiological relevance of organoids, the use of patient-derived organoids has become a useful tool to customize a specific treatment for an individual patient, which is known as personalized medicine (Mun et al. 2019). To apply HuiPSC-derived organoids for personalized medicine, HuiPSCs first need to be generated from a patient's somatic cells. Skin fibroblasts are a primary source for HuiPSC generation, but this involves invasive biopsy of skin (Mun et al. 2019). Second, to form a functioning liver organoid, HuiPSCs must further differentiate into different cell populations, such as the hepatic endoderm, endothelium, and septum mesenchyme [Takebe et al. 2012; Takebe et al. 2013]. Collectively, current methods are complicated and time consuming, limiting their use to a high-throughput setting (Takebe et al. 2013). Here, we developed a simpler method in which the hepatic endoderm alone was able to form liver organoids without coculture with the endothelium and septum mesenchyme. Moreover, this report also demonstrates that hematopoietic progenitor-derived HuiPSCs are 
104 capable of hepatocyte differentiation and liver organoid formation, highlighting the possibility

105 for the use of a less invasive procedure to generate HuiPSCs.

106

107

Materials \& Methods

108

109

Ethical Approval/Declaration: The study research protocol for the use of MUi019 was

110 approved by the Mahidol University Central Institutional Review Board (COA no.

111 038.2803/2016) and the Human Research Protection Unit, Faculty of Medicine Siriraj Hospital, Mahidol University (COA no. Si459/2019).

113

114 Culture of human iPS cells (Si-Tayeb et al. 2010)

115 HuiPS cells (MUi019 line) (Tangprasittipap et al. 2017) were cultured on a cell culture plate 116 coated with $2 \mu \mathrm{g} / \mathrm{mL}$ Matrigel (Growth Factor Reduced; Corning, BD Bioscience). The culture 117 medium for iPS cells consisted of Dulbecco's modified Eagle medium (DMEM) F12, $100 \mu \mathrm{M}$ 118 penicillin/streptomycin (Invitrogen), L-glutamine, $100 \mathrm{ng} / \mathrm{mL}$ fibroblast growth factor 2 (FGF2 119 or bFGF; Invitrogen), $2 \mathrm{ng} / \mathrm{mL}$ TGF $\beta 1,64 \mu \mathrm{g} / \mathrm{mL}$ L-ascorbic acid, and $1 \mathrm{X}$ 120 insulin/transferrin/selenium. Cells were passaged every 5-6 days using 0.5 mM EDTA 121 (Invitrogen) as the cell dissociation solution.

122

Generation of hepatic endoderm, hepatoblasts, and hepatocytes from human iPS cells (SiTayeb et al. 2010)

HuiPS cells were cultured as mentioned above for 5-6 days. Hepatic differentiation consisted of four sequential phases. For the first phase, the culture medium consisted of $100 \mathrm{ng} / \mathrm{mL}$ activin A (R\&D Systems) in RPMI plus 1x B27 (RPMI/B27) medium (Invitrogen) under ambient oxygen $/ 5 \% \mathrm{CO}_{2}$ for 5 days. For the second phase, the culture medium contained $20 \mathrm{ng} / \mathrm{mL}$ bone morphogenetic protein 4 (BMP4, Peprotech) and $10 \mathrm{ng} / \mathrm{mL}$ basic FGF (Invitrogen) in RPMI/B27 medium under $4 \% \mathrm{O}_{2} / 5 \% \mathrm{CO}_{2}$ for 5 days. For the third phase, the culture medium consisted of $20 \mathrm{ng} / \mathrm{mL}$ hepatic growth factor (HGF, Peprotech) in RPMI/B27 medium under 4\% $\mathrm{O}_{2} / 5 \% \mathrm{CO}_{2}$ for 5 days. For the final phase, the cells were cultured with $20 \mathrm{ng} / \mathrm{mL}$ oncostatin-M (R\&D Systems) in RPMI/B27 medium for 5 days and then maintained in ambient oxygen $/ 5 \% \mathrm{CO}_{2}$. 
135 Gene expression analysis

136 RNA was extracted, and cDNA was prepared using a cDNA synthesis kit (Biotechrabbit). The 137 expression of pluripotency-related, endodermal and hepatic genes was assessed using real-time 138 conventional PCR. Primer sets were obtained from previous reports [Schwartz et al. 2002;

139 Takahashi et al. 2007; Chen et al. 2012; Irie et al. 2015; Aguila et al. 2014] and are shown in 140 Table 1. Luna ${ }^{\circledR}$ Universal qPCR Master Mix (New England BioLabs) was used, and the primer 141 concentration was $1 \mu \mathrm{M}$ for each primer. The thermal cycles were as follows: initial denaturation 142 at $95{ }^{\circ} \mathrm{C}$ for $3 \mathrm{~min}, 30$ cycles of denaturation at $95^{\circ} \mathrm{C}$ for $10 \mathrm{~s}$, annealing at $60{ }^{\circ} \mathrm{C}$ for $10 \mathrm{~s}$, and 143 extension at $72{ }^{\circ} \mathrm{C}$ for $10 \mathrm{~s}$, followed by a final extension at $72{ }^{\circ} \mathrm{C}$ for $1 \mathrm{~min}$. Transcript of 144 human actin beta $(A C T B)$ served as internal control to normalize gene expression. Threshold 145 cycles (CT) of each samples were compared with a reference sample using the $2^{-\Delta \Delta \mathrm{CT}}$ method 146 (Rao et al. 2013) and is shown as relative expression. Gene expression analyses were from three 147 independent experiments, and each run of real-time PCR were carried out in triplicate.

\section{Immunofluorescence}

150 The cells or organoids were attached to a glass slide and air-dried. The cells were fixed with $1 \%$ 151 paraformaldehyde in PBS at room temperature for $30 \mathrm{~min}$ and then permeabilized with $0.05 \%$

152 Triton X-100 in PBS for 15 min. After washing with PBS, the cells were exposed to 3\% BSA in 153 PBS for 15 min. Rabbit polyclonal anti-human HNF4 (1:100 from Sigma-Aldrich), mouse 154 monoclonal anti-human $\alpha$-fetoprotein antibody (1:500, Sigma-Aldrich), rabbit polyclonal anti155 human albumin (1:100, Sigma-Aldrich), rabbit polyclonal anti-human CYP3A43 antibody 156 (1:100, Abcam), mouse monoclonal anti-human EpCAM antibody (1:100, GeneTex), rabbit 157 polyclonal anti-human CD31 antibody (1:20, Abcam) and mouse anti-human CD81 (1:200, 158 Abcam) antibodies diluted in 1\% BSA were applied onto the glass slide and incubated overnight. 159 After washing with PBS, the cells were then incubated with Alexa Fluor 594-conjugated goat 160 anti-rabbit IgG and Alexa Fluor 488-conjugated goat anti-mouse IgG antibodies (Invitrogen).

161 The DAPI-containing medium was mounted on the glass slide and covered with a glass 162 coverslip. Images of the cells were visualized using a confocal microscope (ECLIPSE Ti-Clsi4 163 Laser Unit, Nikkon). 
166 Generation of liver organoids from the hepatic endoderm

167 HuiPSC-derived hepatic endoderm was collected from a well of a 24-well plate using cell 168 dissociation buffer (Gibco, LifeTechnologies). The cells were then suspended in a culture 169 medium consisting of $40 \mathrm{ng} / \mathrm{mL}$ HGF and $20 \mathrm{ng} / \mathrm{mL}$ oncostatin M in RPMI/B27. The cell 170 suspension was immersed in ice for $15 \mathrm{~min}$ and immediately mixed with an equal volume of 171 Matrigel. The final mixture contained 50\% Matrigel, $20 \mathrm{ng} / \mathrm{mL}$ HGF (Peprotech), and $17210 \mathrm{ng} / \mathrm{mL}$ oncostatin M. After solidification of the Matrigel at $37^{\circ} \mathrm{C}$ for $15 \mathrm{~min}, \mathrm{RPMI} / \mathrm{B} 27$ 173 medium containing $20 \mathrm{ng} / \mathrm{mL}$ HGF and $10 \mathrm{ng} / \mathrm{mL}$ oncostatin $\mathrm{M}$ was added on top of the solid 174 Matrigel. The cells were cultured under $4 \% \mathrm{O}_{2} / 5 \% \mathrm{CO}_{2}$ at $37^{\circ} \mathrm{C}$. The covering medium was 175 renewed every $48 \mathrm{~h}$. From day 10 onward of 3D culture, the medium on top of Matrigel was 176 changed to the $\mathrm{HCM}^{\mathrm{TM}}$ Hepatocyte Culture Medium BulletKit ${ }^{\mathrm{TM}}$ (herein called HCM) 177 containing $\mathrm{HBM}^{\mathrm{TM}}$ Basal medium and $\mathrm{HCM}^{\mathrm{TM}}$ SingleQuots Supplements, which include 178 transferrin, ascorbic acid, human endothelial growth factor, insulin, hydrocortisone, fatty acid-

179 free BSA and Gentamicin sulfate-Amphotericin. To sub-culture, the plate was place on ice for 10 $180 \mathrm{~min}$. The cold HCM was then added into a well and gently mixed by pipetting to liquefy the 181 Matrigel. A part of liquefied Matrigel was suspended in the cold HCM. An equal volume of the 182 organoid mixture was then mixed with Matrigel and plated into a 96-well plate. After 183 solidification of the Matrigel at $37^{\circ} \mathrm{C}$ for $15 \mathrm{~min}$, the HCM was added on top. The HCM was 184 changed every $48 \mathrm{~h}$.

185

\section{Enzyme-linked immunosorbent assay (ELISA) for human albumin}

Amount of human albumin secreted in culture medium was assessed using the Human Albumin/Serum albumin ELISA kit (Millipore) following manufacturer's instructions. The hepatocyte culture medium was used as background control. Triplicates were performed for each independent experiments.

191

\section{Glycogen accumulation}

193

The cells were treated with 4\% paraformaldehyde in PBS and permeabilized with $0.5 \%$ Triton $\mathrm{X}-100$ in PBS. Cells treated with $1 \mathrm{mg} / \mathrm{mL}$ diastase in PBS (Sigma) were used as the negative control. The cells were then incubated with periodic acid for $5 \mathrm{~min}$, washed with distilled water, 
197 and the nuclei were stained with hematoxylin. After rinse with water, cells were incubated with 198 Bluing reagent for $30 \mathrm{sec}$ followed by incubation with Light Green solution for two min. The 199 glass slide was immersed in absolute alcohol to dehydrate and air-dried.

200

201

202

203

204

205

206

207

208

209

210

211

212

213

214

215

216

217

218

219

220

221

222

223

224

225

226

227

\section{Lipid stain using Oil Red O}

Lipid stored in cells was visualized using fat-soluble Oil Red O following a manufacture' instruction (Oil Red O Stain Kit, Abcam). Cells were attached onto a glass slide using cytocentrifugation. After air dry, the glass slide was placed in propylene glycol for $2 \mathrm{~min}$ and then incubated with Oil Red O solution for 6 min. The glass slide was immersed in $85 \%$ propylene glycol in distilled water for $1 \mathrm{~min}$ and rinsed twice with distilled water. The cells were then stained with Hematoxylin for 1-2 min and rinse thoroughly in tap water. Then, the glass slide was rinsed with two changes of distilled water and air-dried.

\section{Metabolism of indocyanine}

Indocyanine green (ICG) is an organic anion, non-toxic and exclusively eliminated by hepatocytes and has been useful as a marker of hepatocyte. The ICG was added into culture medium at 1 $\mathrm{mg} / \mathrm{mL}$ and incubated at $37^{\circ} \mathrm{C}$ for $60 \mathrm{~min}$. After medium removal, cells were washed with PBS to remove excess extracellular indocyanine.

\section{Statistical analysis}

Data are presented as means \pm standard deviation (SD). Differences were statistically evaluated using Student $t$ test. P-values $<0.05$ were considered statistically significant.

\section{Results}

\section{Hepatic differentiation potential of the HuiPS MUi019 cell line}

The potential of the peripheral blood CD34+ hematopoietic progenitor cell-derived MUi019 cell line for endodermal differentiation has been demonstrated in vivo (Tangprasittipap et al. 2017); however, its capability for hepatic differentiation in vitro is unknown. Here, we first assessed the capability of the HuiPSC MUi019 cell line (Tangprasittipap et al. 2017) for hepatic differentiation. Hierarchically differentiated cells, namely, definitive endoderm, hepatic endoderm (HE), hepatoblasts, and hepatocytes, were prepared according to a method described 
228 in a previous report (Si-Tayeb et al. 2010). Upon exposure to four different sets of cytokines

229 (Fig. 1A), the morphologies of the differentiated cells tended to change in a stepwise fashion

230 (Fig. 1B-1E). On day 25 of differentiation, groups of cells having the same morphology appeared

231 in many focal areas of the cell culture plate (Fig. 1E-1F). Higher magnification observation

232 revealed polygonal cells having large nuclei, which is a main characteristic of hepatocytes (Fig.

$2331 \mathrm{~F})$.

234 To characterize the hepatic cells, mRNA transcripts indicating each cell type were 235 examined using real-time PCR. Here, octamer-binding transcription factor 4 (OCT4) identified 236 an undifferentiated pluripotent stem cells, forkhead box A2 (FOXA2) and sex determining region 237 Y box 17 (SOX17) indicated definitive endoderm. The hepatic endoderm expresses FOXA2.

238 Alpha-fetoprotein $(A F P)$ and albumin $(A L B)$ indicated hepatoblast and mature hepatocytes, 239 respectively. Based on their transcript profile in Fig. 1G-1K, mRNA level of OCT4 decreased as 240 the HuiPSC differentiated into hepatocytes (Fig. 1G). By contrast, that of SOX17 and FOXA2 up241 regulated in the 10-day 2D culture, in which the HuiPSCs specified to hepatic endoderm,

242 respectively; however, level of both transcripts gradually decreased in the 15-day 2D culture 243 during differentiation of hepatic endoderm into hepatoblasts (Fig. 1H and 1I, respectively). Level 244 of $A F P$ transcript was detectable at day 10 until day 25 (Fig. 1J), while that of $A L B$ transcript 245 could be detected at day 25 of 2D culture (Fig. 1K). Collectively, the 25-day-old cells exhibited 246 characteristics of endoderm (SOX17 and FOXA2), hepatoblasts $(A F P)$, and hepatocyte $(A L B)$ 247 cells. In Fig. 1L-1Q, confocal microscopic observations confirmed the presence of intracellularly 248 synthesized albumin (Fig. 1L), a specific marker of hepatic cells, and CD81 (Fig. 1M), an 249 important receptor of the Plasmodium falciparum sporozoite (Silvie et al. 2003) and hepatitis C 250 virus entry (Bruening et al. 2018). Coexistence of albumin and CD81 was also observed 251 (arrowheads in Fig. 1O). Cells stained with $2^{\text {nd }}$ antibody was served as background control (Fig. 252 1P). Representative confocal image revealed $5.9 \pm 1.3 \%$ of the 25 -day-old $2 \mathrm{D}$ cultured cells 253 expressing albumin (Fig. 1Q). The HuiPSC-derived hepatocytes were able to store glycogen 254 (Fig. 1R). Taken together, the results indicated that the HuiPS MUi019 cell line was capable of 255 hepatic cell differentiation in vitro.

256

HuiPSC-derived hepatic endoderm spontaneously formed organoids, but the hepatoblasts 258 and hepatocytes could not 
259 To determine which cell type is capable of forming an organoid, we collected HE, hepatoblasts, 260 and hepatocytes at days 10, 15, and 20 of cell differentiation (Fig. 2A) and cultured them in a 3D 261 setting (50\% Matrigel prepared in RPMI supplemented with 1\% B27, HGF, and oncostatin M)

262 (Fig. 2B). The top of the solidified Matrigel was covered with the same medium described above 263 without Matrigel. On day 5 of the 3D culture, a small, irregularly shaped structure appeared in 264 the culture of the HEs (Fig. 2C), while neither hepatoblasts nor hepatocytes were observed (Fig. 265 2D and 2E, respectively). Therefore, 2D-cultured, 10-day-old HE was used further for organoid 266 formation. On day 7 of the 3D culture, irregularly shaped cell clumps composed of an outer layer 267 and inner mass could be clearly observed under a microscope. The outer layer had a protruding, 268 spike-like structure (Fig. 2F-2G). By day 10 of the 3D culture, the sizes of cell clumps increased, 269 while their pikes disappeared, and the outer layer became smooth (Fig. 2H-2I). At this time 270 point, the organoid showed the characteristics of a spherical, hollow shape, hereafter called a 271 hepatic endoderm organoid or HEO. From day 10 onward of the 3D culture, the covering 272 medium was changed to the HCM for hepatocyte maturation.

273

\section{Characterization of the HuiPS-derived HEOs}

275 On day 12 of the 3D culture, the HEO had space at the center surrounded by a layer of cells, 276 which are characteristics typical of a blastocyst (Fig. 3A). The sizes of the HEOs varied and 277 increased from day 12 onward (Fig. 3B-3C). Despite their increasing sizes, there was no fusion 278 of adjacent HEOs. Instead, the outer layer of the HEO was bent (Fig. 3B and 3C, arrowheads). 279 Microscopic observation of the HEOs on days 12 and 14 revealed a thin, single-layer structure 280 (Fig. 3D and 3E, respectively). By day 17, dome-shaped cells appeared (Fig. 3F, arrowheads). 281 Higher magnification observation of the 17-day HEO yielded microscopic images showing a 282 very thin layer (Fig. 3G). When focusing on the cells, polygonal cells could be observed 283 (arrowheads in Fig. 3H).

284 To characterize the 17-day-old HEOs, gene and protein expression analyses were 285 performed. Although the HEOs did express the pluripotency-related OCT4 (Fig. 1G) and the 286 endoderm-specific transcription factor $S O X 17$ (Fig. 1H); however, the level was much lower 287 than the 2D culture. By contrast, the 17-day-old HEOs expressed hepatic transcription factor $288 F O X A 2$ (Fig. 1I), hepatoblast-specific factor $A F P$ (Fig. 1J), and hepatocyte-specific $A L B$ (Fig. $2891 \mathrm{~K})$ at higher level compaired to the 25-day-old 2D-cultured hepatocyte, thus implying the 
290 greater maturation of the 3D culture. Then, we evaluated the expression of hepatocyte-related 291 proteins in a time course manner using immunofluorescence and confocal microscopy. The 292 confocal images revealed expression of hepatic nuclear factor 4 (HNF4) at both day 12 (Fig. 3I293 3K) and 17 (Fig. 3L-3N) of the 3D culture. Moreover, the confocal images also confirmed that 294 the $A L B$ transcripts were translated into albumin (Fig. 3O-3T), As shown in Fig. 3U-3Z, the 295 HEOs expressed AFP at day 12 and 17 of the 3D culture. Although CYP4A3 was not observed at 296 the 12-day-old HEOs, CYP4A4 was subsequently detected at day 17 of the 3D culture (Fig. 297 3AA-3FF). To ensure that HEO could be applied as a model of infectious diseases, we examined 298 the expression of CD81, an important receptor for Plasmodium falciparum sporozoites and 299 hepatitis C virus. As shown in Fig. 3GG-3LL, the 12-day- and 17-day-old HEOs expressed 300 CD81. Localization of albumin and CD81 was performed. Albumin was expressed in an 301 asymmetric pattern (Fig. 3MM-3PP); however, the expression of CD81 overlapped with that of 302 albumin in some areas of the HEOs (Fig. 3PP). Higher magnification images revealed the 303 coexpression of albumin and CD81 (Fig. 3QQ-3TT). Based on cell counting, higher percentage 304 of ALB+ cells was observed in the HEOs (bar graph in Fig 1Q). All confocal images were 305 compared with the HEOs stained with the $2^{\text {nd }}$ antibody (Fig. 3UU-3XX). In comparable to 306 immunofluorescence, the 17-day-old HEOs secreted albumin into the culture medium at $1,077 \pm 176 \mathrm{ng}$ per $10^{6}$ examined cells within $48 \mathrm{~h}$ of culture (Fig. 3YY). By contrast, among three independent experiments, the 25-day-old 2D cultured hepatic cells secreted albumin into the culture medium at $44.9 \pm 53 \mathrm{ng}$ per $10^{6}$ cells within $48 \mathrm{~h}$ of culture. Apart from the immunofluorescence, both day 12 and 17-derived HEOs were capable of storing glycogen (Fig. $3 \mathrm{ZZ}$ and 3AAA) and lipid (Fig. 3BBB-3CCC) and metabolizing indocyanine (Fig. 3DDD-3EEE)

312 in a time-course manner. Collectively, the data of gene and protein expression imply a full 313 maturation of the HEOs within 17 days of 3D culture, which is equivalent to the 27-day period. Given the high cost of Matrigel, we next determined the minimum concentration of Matrigel in which the 3D structure of the organoid remained unchanged. The 17-day-old organoids were split by dilution with the medium to obtain $25 \%, 12.5 \%$, and $6.25 \%$ Matrigel prepared in RPMI/B27 supplemented with HGF and oncostatin M (Fig. 4A). Regardless of the Matrigel 
321 intact. In our experience, using Matrigel at a concentration lower than $6.25 \%$ leads to difficulty

322 in changing the upper liquid medium during long-term culture.

323

324

\section{Long-term culture of the HEOs}

325 We further evaluated whether the HEOs could be maintained hepatocyte characteristics in a 326 long-term culture. The HEOs were sub-culture weekly and the culture continued for 60 days in total. Representative images of a well of 96-well plate show morphology and size of the 60-dayold HEOs under a microscope after a week of sub-culture (Fig. 5A). At higher magnification, the HEOs remain single layer of cells (Fig. 5B) having a polygonal shape plus large nuclei as well as binucleated cell (respective white and black arrowhead in Fig. 5C), characteristics of hepatic cells. Confocal images of the day 60-derived HEOs showing the expression of hepatocytespecific ALB (Fig. 5D-5F), CYP4A3 (Fig. 5G-5I) and CD81 (Fig. 5J-5L). To clearly demonstrate, higher magnifications of the images are shown in Fig. 5M-5U. In agreement with immunofluorescence, the 60-day-old HEOs secreted albumin at 1,106 $\pm 77 \mathrm{ng}$ per $10^{6}$ cells within $48 \mathrm{~h}$, a similar level to the 17-day-old HEOs (Fig. 3YY). By contrast, when we maintained the 2D culture of the HuiPSC-derived hepatic cells for 40 days, lower amount of albumin could be detected relative to that of the day 25-derived hepatic cells (Fig. 3YY), implying loss of albumin synthesis an in vitro culture. Moreover, the day 60-derived HEOs were still able to storing glycogen (Fig. 5V) and lipid (Fig. 5W), and metabolizing indocyanine (Fig. 5X) as well as expressing hepatic stem/progenitor marker EpCM (Fig. 5Y-5AA) but not endothelial marker CD31 (Fig. 5BB-5DD). Taken together, the HEOs remained characteristics of hepatic cells after long-term culture in a Matrigel.

\section{Discussion}

345 Here, we developed a simple and fast protocol to generate a functioning human liver organoid 346 from pluripotent stem cells derived from peripheral blood CD34+ cells. By day 15 of the initial 347 culture of HuiPSCs, the HEOs had developed and required an additional 2-3 days to synthesize 348 albumin, an indicator of a functioning hepatocyte. To the best of our knowledge, the protocol 349 used in this study is faster than that reported in a recent study (Mun et al. 2019) and does not 350 require a mixture of endothelial cells and mesenchymal cells [Takebe et al. 2012; Takebe et al. 351 2013], endothelial cells (Pettinato et al. 2019), or hepatic progenitor cells ( $\mathrm{Ng}$ et al. 2018) as 
352 starting components. In agreement with the results of other studies, the spherical structures of

353 liver organoids were also reportedly observed [Huch et al. 2015; Mun et al. 2019].

354 Several studies have attempted to generate fully functioning liver organoids. Coculture of

355 HuiPSCs with adipose microvascular endothelial cells in 3D culture could form liver organoids

356 (Pettinato et al. 2019). iPS cell-derived hepatic progenitors reportedly formed liver organoids

357 using an inverted colloid crystal, which provided mechanical properties to recapitulate the

358 extracellular niche ( $\mathrm{Ng}$ et al. 2018). Moreover, spherical structures emerged from a 2D culture

359 monolayer of HuiPSCs that were capable of forming liver organoids in liquid and semisolid

360 cultures; however, it took approximately 22 days to obtain the spherical structures, and

361 additional 8-9 days as well as two stepwise medium were needed for maturation (Mun et al.

362 2019). Compared to the results of these reports, the advantages of our protocol, in which mature

363 hepatocyte could be obtained by day 27 of HuiPSC culture, include simplicity and rapidness

364 [Takebe et al. 2013; Takebe et al. 2012; $\mathrm{Ng}$ et al. 2018; Pettinato et al. 2019] . Nevertheless,

365 there remains a drawback in our protocol. Given a lack of comparison with primary hepatocyte

366 culture, how extent maturation of hepatocytes in the 27-day-old HEOs is unknown. Thus, we

367 indirectly compared amount of secreted albumin in culture of primary hepatocyte $(1,500-1,700$

$368 \mathrm{ng} / \mathrm{mL} /$ day $/ 10^{6}$ cells) (Mun et al. 2019) with the 27-day-old HEOs $\left(1,077 \pm 176 \mathrm{ng} / 48 \mathrm{~h} / 10^{6}\right.$ cells).

369 The comparison implies that the 27-day-old HEOs were immature relative to the 2D culture of

370 primary hepatocytes. In agreement, expression of EpCAM, which mature hepatocytes are devoid

371 of (Dolle et al. 2015), was observed in most cells of the 27-day-old HEOs (Fig. 5AA).

372 Therefore, lower extent of maturation was likely a limitation of our protocol.

373 iPS cells can be generated from various tissue sources, including fibroblasts from skin

374 [Alawad et al. 2016; Du et al. 2015] and hematopoietic cells [Haase et al. 2017; Takenaka et al.

375 2010; Kim et al. 2013] or lymphocytes (Phillips et al. 2012) from peripheral blood, and have

376 been used in disease modeling. HuiPSC lines derived from peripheral blood T lymphocytes

377 reportedly differentiate into hepatocytes more efficiently than hiPSC clones derived from adult

378 dermal fibroblasts (Kajiwara et al. 2012). To the best of our knowledge, this study is the first

379 report showing that CD34+ cell-derived iPS cells could differentiate into hepatocytes and hepatic

380 endoderm with the ability to give rise to liver organoids. Collecting peripheral blood is less

381 invasive than skin biopsy; thus, PB has become the first choice for the generation of PSCs.

382 Therefore, our protocol is probably suitable for specific and safe personalized therapy for 
383 hepatotropic diseases. To ensure that our protocol could be used to generate a model of

384 hepatotropic diseases, we therefore examined the expression of the pathogen entry receptor. The 385 iPS-derived liver organoids expressed CD81 (Foquet et al. 2015) (Silvie et al. 2006) (Silvie et al. 386 2003; Yalaoui et al. 2008), a potent cell receptor allowing Plasmodium sporozoite invasion into 387 hepatocytes. Thus, this would allow the examination of host-pathogen interactions in a model 388 that is physiologically relevant to the human body. Regarding the clinical application of liver 389 organoids in transplantation, it is necessary to demonstrate further that the HEOs generated from 390 this study are capable of rescuing animals affected by lethal hepatic diseases.

391

392 Conclusions

393 CD34+ cell-derived HuiPSCs were capable of differentiating into hepatocytes and hepatic

394 endoderm with the ability to give rise to liver organoids. 3D culture of the hepatic endoderm is a 395 relatively fast, simple, and less laborious way to generate liver organoids from HuiPSCs that is more physiologically relevant than 2D culture. Further examination of host-pathogen interactions in this HE-derived liver organoids is necessary.

\section{Acknowledgements}

400

The authors gratefully acknowledge Prof. Dr. Wanpen Chaicumpa for valuable scientific suggestion, and the Institute of Molecular Biosciences, the Siriraj Central Research Facility and Department of Anatomy, Faculty of Medicine Siriraj Hospital, Mahidol University for technical 403 support.

Figure 1 Hepatic differentiation potential of the HuiPSC MUi019 line. (A) Schematic 406 diagram of hepatic cell differentiation in the $2 \mathrm{D}$ culture system. The protocol consisted of four stepwise phases: endoderm, hepatic endoderm, hepatoblast, and hepatocyte. Cells were cultured under $5 \%$ or $20 \% \mathrm{O}_{2}$. (B-E) Representative microscopic bright-field images of differentiated iPS cells after exposure to different sets of cytokines (20X objective lens). (F) Microscopic image of 25-day-old differentiated cells at higher magnification. The dotted line of the left panel indicates the area where polygonal cells 412 having large nuclei were observed. (G-K) Gene expression profile of cells at different 413 stages of culture. Relative expression levels were calculated using the $2^{-\Delta \Delta C T}$ method. 414 Data are the mean $\pm S D(n=3)$ and statistically analyzed using Student's $t$ test. (L-P) 
415 Confocal microscopic observation of albumin (red color) and CD81 (green color). Scale

416 bar $=50 \mu \mathrm{m}$. (Q) Percentage of albumin-expressing cells observed using

417 immunofluorescence and confocal microscopy. (R) Glycogen storage in the 25-day

418 differentiated cells from E. Representative image shown was obtained with a 100X

419 objective lens. Light microscopic and confocal images are representative of three

420 independent experiments.

421

422 Figure 2 Organoid formation potential of the hepatic endoderm, hepatoblast, and 423 hepatocyte phases. (A) Schematic diagram of the methods used for assessing organoid 424 formation ability. Hepatic endoderm (day 10), hepatoblasts (day 15), and hepatocytes 425 (day 20) were cultured in semisolid Matrigel (pink color). Liquid medium (light orange) 426 was added on top of the Matrigel. (C-E) Ability of hepatic endoderm, hepatoblasts, and 427 hepatocytes to form an organoid in the Matrigel-based 3D culture was assessed at day 5 428 of the 3D culture. Images shown were obtained with a $40 \mathrm{X}$ objective lens. (F-G) Two 429 representative images of the organoid in the Matrigel-based 3D culture at day 7 . (H-I) 430 Two representative images of the organoid in the Matrigel-based 3D culture at day 10. 431 Three independent experiments were performed and representative microscopic images 432 were shown.

433

434 Figure 3 Morphology of hepatic endoderm-derived organoids (HEOs). (A-C) 435 Morphology of the HEOs at days 12, 14, and 17 postculture in Matrigel (4X objective 436 lens). (D-F): Representative images of single HEOs are shown at high magnification 437 (40X). (G) The outer layer of cells is shown. (H) Zoomed-in image of the HEOs showing 438 the cells with a polygonal shape and large nuclei (arrowheads). (I-LL) Confocal image of 439 a day 17-derived organoid showing the expression of hepatic nuclear factor 4 (HNF4), 440 hepatocyte-specific albumin (ALB) and $\alpha$-fetoprotein (AFP), cytochrome P450 3A4 441 (CYP4A3), and CD81, an important receptor of Plasmodium falciparum sporozoites. (MM442 PP) Co-localization of CD81 and ALB in the 17-day HEO. The confocal images of 443 organoid show the expression of CD81 (green) and hepatocyte-specific albumin (red). 444 (QQ-TT) Zoomed-in images reveal the albumin- and CD81-expressing cells. (UU-XX) 445 Cells stained with $2^{\text {nd }}$ antibody specific to $\lg G$ of rabbit and mouse (unstained samples) 
446 served as background control. Four independent experiments were performed and 447 representative images were shown. (YY) Total amount of human albumin secreted within $44848 \mathrm{~h}$ in the culture medium of the 25 - and 40 -day 2D culture and the 17 - and 60 -day $3 \mathrm{D}$ 449 culture. Total amount (ng) of human albumin in each experiments were calculated based 450 on number of cells. Individuals represent independent experiments. Data are the mean \pm 451 SD ( $n=3-4)$ and statistically analyzed using Student's $t$ test. Culture medium of 452 undifferentiated HuiPSCs was used for comparison. (ZZ-EEE) Hepatocyte functions 453 (glycogen and lipid storage, and metabolism of indocyanine) of a day 12- and 17-derived 454 organoids. Four independent experiments were performed and representative images 455 were shown.

Figure 4 Minimal concentration of Matrigel needed to maintain the 3D structure of

458

459

460

461

462

463

464

465

466

467

468

469

470

471

472

473

474

475

the organoid. A: Schematic diagram showing a method used to determine the minimum concentration of Matrigel in which the 3D structure of the organoid could be maintained; B: Microscopic images of the liver organoid. The 20-day-old organoids were split to obtain $25 \%, 12.5 \%$, and $6.25 \%$ Matrigel in RPMI/B27, which were supplemented with HGF and cultured for 5 days. All images were captured using a 20X objective lens. Three independent experiments were performed and representative images were shown.

Figure 5 Long-term maintenance of the hepatic endoderm-derived organoid. (A) Morphology of the HEOs at days 60 postculture in Matrigel. Representative images of a well of 96-well plate show the HEOs after a week of sub-culture. (B) At higher magnification, the outer layer of cells remain single. (C) Focused image of the HEOs showing the cells with a polygonal shape and large nuclei (white arrowhead) as well as binucleated cell (black arrowhead), characteristics of hepatic cells. (D-L) Confocal images of the day 60-derived HEOs showing the expression of hepatocyte-specific albumin (ALB), cytochrome P450 3A4 (CYP4A3) and CD81. (M-U) Zoomed-in confocal images shown in (D). (V-X) Hepatocyte functions (glycogen and lipid storage, and metabolism of indocyanine) of a day 60-derived organoids. (Y-AA) Confocal images of the day 60derived HEOs showing the expression of EpCAM. (BB-DD) Confocal images of the day 
476

477

478

479

480

481

482

483

484

485

486

487

488

489

490

491

492

493

494

495

496

497

498

499

500

501

502

503

504

505

506

507

508

509

510

511

512

513

514

515

516

517

518

519

520

521

522

523

60-derived HEOs showing the expression of CD31. Four independent experiments were performed and representative images were shown.

\section{References}

Aguila JC, Blak A, van Arensbergen J, Sousa A, Vazquez N, Aduriz A, Gayosso M, Lopez Mato MP, Lopez de Maturana R, Hedlund E, Sonntag KC, and Sanchez-Pernaute R. 2014.

Selection Based on FOXA2 Expression Is Not Sufficient to Enrich for Dopamine Neurons From Human Pluripotent Stem Cells. Stem Cells Transl Med 3:1032-1042. 10.5966/sctm.2014-0011

Alawad A, Alhazzaa O, Altuwaijri S, Alkhrayef M, Alagrafi F, Alhamdan Z, Alenazi A, Alharbi S, and Hammad M. 2016. Generation of human iPS cell line SKiPSc1 from healthy Human Neonatal Foreskin Fibroblast cells. Stem Cell Res 17:158-160. 10.1016/j.scr.2016.06.006

Bartfeld S, Bayram T, van de Wetering M, Huch M, Begthel H, Kujala P, Vries R, Peters PJ, and Clevers H. 2015. In vitro expansion of human gastric epithelial stem cells and their responses to bacterial infection. Gastroenterology 148:126-136 e126. 10.1053/j.gastro.2014.09.042

Boj SF, Hwang CI, Baker LA, Chio, II, Engle DD, Corbo V, Jager M, Ponz-Sarvise M, Tiriac H, Spector MS, Gracanin A, Oni T, Yu KH, van Boxtel R, Huch M, Rivera KD, Wilson JP, Feigin ME, Ohlund D, Handly-Santana A, Ardito-Abraham CM, Ludwig M, Elyada E, Alagesan B, Biffi G, Yordanov GN, Delcuze B, Creighton B, Wright K, Park Y, Morsink FH, Molenaar IQ, Borel Rinkes IH, Cuppen E, Hao Y, Jin Y, Nijman IJ, lacobuzioDonahue C, Leach SD, Pappin DJ, Hammell M, Klimstra DS, Basturk O, Hruban RH, Offerhaus GJ, Vries RG, Clevers H, and Tuveson DA. 2015. Organoid models of human and mouse ductal pancreatic cancer. Cell 160:324-338. 10.1016/j.cell.2014.12.021

Bruening J, Lasswitz L, Banse P, Kahl S, Marinach C, Vondran FW, Kaderali L, Silvie O, Pietschmann T, Meissner F, and Gerold G. 2018. Hepatitis C virus enters liver cells using the CD81 receptor complex proteins calpain-5 and CBLB. PLoS Pathog 14:e1007111. 10.1371/journal.ppat.1007111

Castellanos-Gonzalez A, Cabada MM, Nichols J, Gomez G, and White AC, Jr. 2013. Human primary intestinal epithelial cells as an improved in vitro model for Cryptosporidium parvum infection. Infect Immun 81:1996-2001. 10.1128/IAI.01131-12

Chen YF, Tseng CY, Wang HW, Kuo HC, Yang VW, and Lee OK. 2012. Rapid generation of mature hepatocyte-like cells from human induced pluripotent stem cells by an efficient three-step protocol. Hepatology 55:1193-1203. 10.1002/hep.24790

Cugola FR, Fernandes IR, Russo FB, Freitas BC, Dias JL, Guimaraes KP, Benazzato C, Almeida N, Pignatari GC, Romero S, Polonio CM, Cunha I, Freitas CL, Brandao WN, Rossato C, Andrade DG, Faria Dde P, Garcez AT, Buchpigel CA, Braconi CT, Mendes E, Sall AA, Zanotto PM, Peron JP, Muotri AR, and Beltrao-Braga PC. 2016. The Brazilian Zika virus strain causes birth defects in experimental models. Nature 534:267271. 10.1038/nature18296

Dekkers JF, Wiegerinck CL, de Jonge HR, Bronsveld I, Janssens HM, de Winter-de Groot KM, Brandsma AM, de Jong NW, Bijvelds MJ, Scholte BJ, Nieuwenhuis EE, van den Brink S, Clevers H, van der Ent CK, Middendorp S, and Beekman JM. 2013. A functional CFTR assay using primary cystic fibrosis intestinal organoids. Nat Med 19:939-945. 10.1038/nm.3201 
524

525

526

527

528

529

530

531

532

533

534

535

536

537

538

539

540

541

542

543

544

545

546

547

548

549

550

551

552

553

554

555

556

557

558

559

560

561

562

563

564

565

566

567

568

569

570

571

572

573

574

Dolle L, Theise ND, Schmelzer E, Boulter L, Gires O, and van Grunsven LA. 2015. EpCAM and the biology of hepatic stem/progenitor cells. Am J Physiol Gastrointest Liver Physiol 308:G233-250. 10.1152/ajpgi.00069.2014

Du SH, Tay JC, Chen C, Tay FC, Tan WK, Li ZD, and Wang S. 2015. Human iPS cell-derived fibroblast-like cells as feeder layers for iPS cell derivation and expansion. J Biosci Bioeng 120:210-217. 10.1016/j.jbiosc.2014.12.009

Farin HF, Karthaus WR, Kujala P, Rakhshandehroo M, Schwank G, Vries RG, Kalkhoven E, Nieuwenhuis EE, and Clevers H. 2014. Paneth cell extrusion and release of antimicrobial products is directly controlled by immune cell-derived IFN-gamma. J Exp Med 211:1393-1405. 10.1084/jem.20130753

Finkbeiner SR, Zeng XL, Utama B, Atmar RL, Shroyer NF, and Estes MK. 2012. Stem cellderived human intestinal organoids as an infection model for rotaviruses. MBio 3:e00159-00112. 10.1128/mBio.00159-12

Foquet L, Hermsen CC, Verhoye L, van Gemert GJ, Cortese R, Nicosia A, Sauerwein RW, Leroux-Roels G, and Meuleman P. 2015. Anti-CD81 but not anti-SR-BI blocks Plasmodium falciparum liver infection in a humanized mouse model. $J$ Antimicrob Chemother 70:1784-1787. 10.1093/jac/dkv019

Forbester JL, Goulding D, Vallier L, Hannan N, Hale C, Pickard D, Mukhopadhyay S, and Dougan G. 2015. Interaction of Salmonella enterica Serovar Typhimurium with Intestinal Organoids Derived from Human Induced Pluripotent Stem Cells. Infect Immun 83:29262934. 10.1128/IAI.00161-15

Gao D, Vela I, Sboner A, laquinta PJ, Karthaus WR, Gopalan A, Dowling C, Wanjala JN, Undvall EA, Arora VK, Wongvipat J, Kossai M, Ramazanoglu S, Barboza LP, Di W, Cao Z, Zhang QF, Sirota I, Ran L, MacDonald TY, Beltran H, Mosquera JM, Touijer KA, Scardino PT, Laudone VP, Curtis KR, Rathkopf DE, Morris MJ, Danila DC, Slovin SF, Solomon SB, Eastham JA, Chi P, Carver B, Rubin MA, Scher HI, Clevers H, Sawyers $\mathrm{CL}$, and Chen Y. 2014. Organoid cultures derived from patients with advanced prostate cancer. Cell 159:176-187. 10.1016/j.cell.2014.08.016

Garcez PP, Loiola EC, Madeiro da Costa R, Higa LM, Trindade P, Delvecchio R, Nascimento JM, Brindeiro R, Tanuri A, and Rehen SK. 2016. Zika virus impairs growth in human neurospheres and brain organoids. Science 352:816-818. 10.1126/science.aaf6116

Haase A, Gohring G, and Martin U. 2017. Generation of non-transgenic iPS cells from human cord blood CD34(+) cells under animal component-free conditions. Stem Cell Res 21:7173. 10.1016/j.scr.2017.03.022

Hubert CG, Rivera M, Spangler LC, Wu Q, Mack SC, Prager BC, Couce M, McLendon RE, Sloan AE, and Rich JN. 2016. A Three-Dimensional Organoid Culture System Derived from Human Glioblastomas Recapitulates the Hypoxic Gradients and Cancer Stem Cell Heterogeneity of Tumors Found In Vivo. Cancer Res 76:2465-2477. 10.1158/00085472.CAN-15-2402

Huch M, Gehart H, van Boxtel R, Hamer K, Blokzijl F, Verstegen MM, Ellis E, van Wenum M, Fuchs SA, de Ligt J, van de Wetering M, Sasaki N, Boers SJ, Kemperman $\mathrm{H}$, de Jonge J, ljzermans JN, Nieuwenhuis EE, Hoekstra R, Strom S, Vries RR, van der Laan LJ, Cuppen E, and Clevers H. 2015. Long-term culture of genome-stable bipotent stem cells from adult human liver. Cell 160:299-312. 10.1016/j.cell.2014.11.050

Irie N, Weinberger L, Tang WW, Kobayashi T, Viukov S, Manor YS, Dietmann S, Hanna JH, and Surani MA. 2015. SOX17 is a critical specifier of human primordial germ cell fate. Cell 160:253-268. 10.1016/j.cell.2014.12.013

Kajiwara M, Aoi T, Okita K, Takahashi R, Inoue H, Takayama N, Endo H, Eto K, Toguchida J, Uemoto S, and Yamanaka S. 2012. Donor-dependent variations in hepatic differentiation from human-induced pluripotent stem cells. Proc Natl Acad Sci U S A 109:12538-12543. 10.1073/pnas.1209979109

Peer) reviewing PDF | (2020:02:45512:1:2:NEW 1 Aug 2020) 
575

576

577

578

579

580

581

582

583

584

585

586

587

588

589

590

591

592

593

594

595

596

597

598

599

600

601

602

603

604

605

606

607

608

609

610

611

612

613

614

615

616

617

618

619

620

621

622

623

624

Kim EM, Manzar G, and Zavazava N. 2013. Human iPS cell-derived hematopoietic progenitor cells induce T-cell anergy in in vitro-generated alloreactive CD8(+) T cells. Blood 121:5167-5175. 10.1182/blood-2012-11-467753

Lancaster MA, Renner M, Martin CA, Wenzel D, Bicknell LS, Hurles ME, Homfray T, Penninger JM, Jackson AP, and Knoblich JA. 2013. Cerebral organoids model human brain development and microcephaly. Nature 501:373-379. 10.1038/nature12517

Leslie JL, Huang S, Opp JS, Nagy MS, Kobayashi M, Young VB, and Spence JR. 2015. Persistence and toxin production by Clostridium difficile within human intestinal organoids result in disruption of epithelial paracellular barrier function. Infect Immun 83:138-145. 10.1128/IAI.02561-14

Mun SJ, Ryu JS, Lee MO, Son YS, Oh SJ, Cho HS, Son MY, Kim DS, Kim SJ, Yoo HJ, Lee HJ, Kim J, Jung CR, Chung KS, and Son MJ. 2019. Generation of expandable human pluripotent stem cell-derived hepatocyte-like liver organoids. J Hepatol 71:970-985. 10.1016/j.jhep.2019.06.030

Ng SS, Saeb-Parsy K, Blackford SJI, Segal JM, Serra MP, Horcas-Lopez M, No DY, Mastoridis S, Jassem W, Frank CW, Cho NJ, Nakauchi H, Glenn JS, and Rashid ST. 2018. Human iPS derived progenitors bioengineered into liver organoids using an inverted colloidal crystal poly (ethylene glycol) scaffold. Biomaterials 182:299-311. 10.1016/j.biomaterials.2018.07.043

Pettinato G, Lehoux S, Ramanathan R, Salem MM, He LX, Muse O, Flaumenhaft R, Thompson MT, Rouse EA, Cummings RD, Wen X, and Fisher RA. 2019. Generation of fully functional hepatocyte-like organoids from human induced pluripotent stem cells mixed with Endothelial Cells. Sci Rep 9:8920. 10.1038/s41598-019-45514-3

Phillips MJ, Wallace KA, Dickerson SJ, Miller MJ, Verhoeven AD, Martin JM, Wright LS, Shen W, Capowski EE, Percin EF, Perez ET, Zhong X, Canto-Soler MV, and Gamm DM. 2012. Blood-derived human iPS cells generate optic vesicle-like structures with the capacity to form retinal laminae and develop synapses. Invest Ophthalmol Vis Sci 53:2007-2019. 10.1167/iovs.11-9313

Rao X, Huang X, Zhou Z, and Lin X. 2013. An improvement of the $2^{\wedge}$ (-delta delta CT) method for quantitative real-time polymerase chain reaction data analysis. Biostat Bioinforma Biomath 3:71-85.

Sato T, Vries RG, Snippert HJ, van de Wetering M, Barker N, Stange DE, van Es JH, Abo A, Kujala P, Peters PJ, and Clevers H. 2009. Single Lgr5 stem cells build crypt-villus structures in vitro without a mesenchymal niche. Nature 459:262-265. 10.1038/nature07935

Schwartz RE, Reyes M, Koodie L, Jiang Y, Blackstad M, Lund T, Lenvik T, Johnson S, Hu WS, and Verfaillie CM. 2002. Multipotent adult progenitor cells from bone marrow differentiate into functional hepatocyte-like cells. J Clin Invest 109:1291-1302. 10.1172/JCI15182

Si-Tayeb K, Noto FK, Nagaoka M, Li J, Battle MA, Duris C, North PE, Dalton S, and Duncan SA. 2010. Highly efficient generation of human hepatocyte-like cells from induced pluripotent stem cells. Hepatology 51:297-305. 10.1002/hep.23354

Silvie O, Greco C, Franetich JF, Dubart-Kupperschmitt A, Hannoun L, van Gemert GJ, Sauerwein RW, Levy S, Boucheix C, Rubinstein E, and Mazier D. 2006. Expression of human CD81 differently affects host cell susceptibility to malaria sporozoites depending on the Plasmodium species. Cell Microbiol 8:1134-1146. 10.1111/j.14625822.2006.00697.x

Silvie O, Rubinstein E, Franetich JF, Prenant M, Belnoue E, Renia L, Hannoun L, Eling W, Levy S, Boucheix C, and Mazier D. 2003. Hepatocyte CD81 is required for Plasmodium falciparum and Plasmodium yoelii sporozoite infectivity. Nat Med 9:93-96. $10.1038 / \mathrm{nm} 808$

Peer] reviewing PDF | (2020:02:45512:1:2:NEW 1 Aug 2020) 
625

626

627

628

629

630

631

632

633

634

635

636

637

638

639

640

641

642

643

644

645

646

647

648

649

650

651

652

653

654

Takahashi K, Tanabe K, Ohnuki M, Narita M, Ichisaka T, Tomoda K, and Yamanaka S. 2007. Induction of pluripotent stem cells from adult human fibroblasts by defined factors. Cell 131:861-872. 10.1016/j.cell.2007.11.019

Takebe T, Sekine K, Enomura M, Koike H, Kimura M, Ogaeri T, Zhang RR, Ueno Y, Zheng YW, Koike N, Aoyama S, Adachi Y, and Taniguchi H. 2013. Vascularized and functional human liver from an iPSC-derived organ bud transplant. Nature 499:481-484. 10.1038/nature12271

Takebe T, Sekine K, Suzuki Y, Enomura M, Tanaka S, Ueno Y, Zheng YW, and Taniguchi H. 2012. Self-organization of human hepatic organoid by recapitulating organogenesis in vitro. Transplant Proc 44:1018-1020. 10.1016/j.transproceed.2012.02.007

Takenaka C, Nishishita N, Takada N, Jakt LM, and Kawamata S. 2010. Effective generation of iPS cells from CD34+ cord blood cells by inhibition of p53. Exp Hematol 38:154-162. 10.1016/j.exphem.2009.11.003

Tangprasittipap A, Jittorntrum B, Wongkummool W, Kitiyanant N, and Tubsuwan A. 2017. Generation of induced pluripotent stem cells from peripheral blood CD34+ hematopoietic progenitors of a 31year old healthy woman. Stem Cell Res 20:91-93. 10.1016/j.scr.2017.02.013

Xia Y, Nivet E, Sancho-Martinez I, Gallegos T, Suzuki K, Okamura D, Wu MZ, Dubova I, Esteban CR, Montserrat N, Campistol JM, and Izpisua Belmonte JC. 2013. Directed differentiation of human pluripotent cells to ureteric bud kidney progenitor-like cells. Nat Cell Biol 15:1507-1515. 10.1038/ncb2872

Yalaoui S, Zougbede S, Charrin S, Silvie O, Arduise C, Farhati K, Boucheix C, Mazier D, Rubinstein E, and Froissard P. 2008. Hepatocyte permissiveness to Plasmodium infection is conveyed by a short and structurally conserved region of the CD81 large extracellular domain. PLoS Pathog 4:e1000010. 10.1371/journal.ppat.1000010

Zomer-van Ommen DD, Pukin AV, Fu O, Quarles van Ufford LH, Janssens HM, Beekman JM, and Pieters RJ. 2016. Functional Characterization of Cholera Toxin Inhibitors Using Human Intestinal Organoids. J Med Chem 59:6968-6972. 10.1021/acs.jmedchem.6b00770

Peer] reviewing PDF | (2020:02:45512:1:2:NEW 1 Aug 2020) 


\section{Figure 1}

Hepatic differentiation potential of the HuiPSC MUi019 line.

(A) Schematic diagram of hepatic cell differentiation in the 2D culture system. The protocol consisted of four stepwise phases: endoderm, hepatic endoderm, hepatoblast, and hepatocyte. Cells were cultured under $5 \%$ or $20 \% \mathrm{O}_{2}$. (B-E) Representative microscopic bright-field images of differentiated iPS cells after exposure to different sets of cytokines (20X objective lens). (F) Microscopic image of 25-day-old differentiated cells at higher magnification. The dotted line indicates the area where polygonal cells having large nuclei were observed. (G-K) Gene expression profile of cells at different stages of culture. Relative expression levels were calculated using the $2^{-\Delta C T}$ method. Data are the mean $\pm S D(n=3)$ and statistically analyzed using Student's t test. (L-P) Confocal microscopic observation of albumin (red color) and CD81 (green color). Scale bar $=50 \mathrm{~mm}$. (Q) Percentage of albuminexpressing cells observed using immunofluorescence and confocal microscopy. (R) Glycogen storage in the 25-day differentiated cells from E. Representative image shown was obtained with a 100X objective lens. Light microscopic and confocal images are representative of three independent experiments. 
A

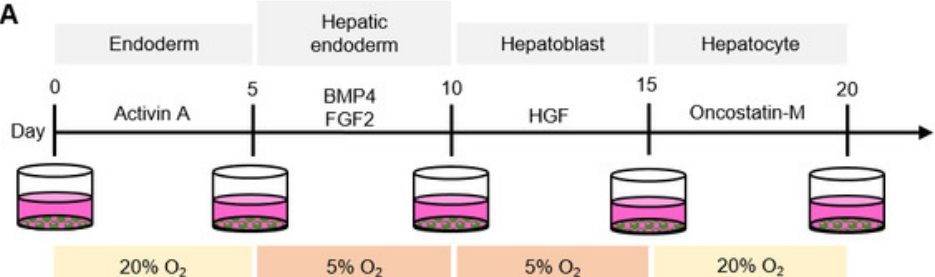

B

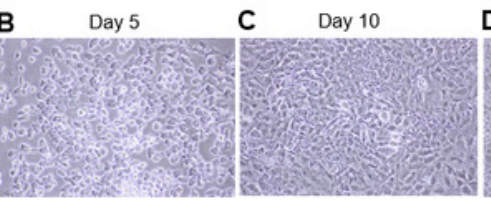

F
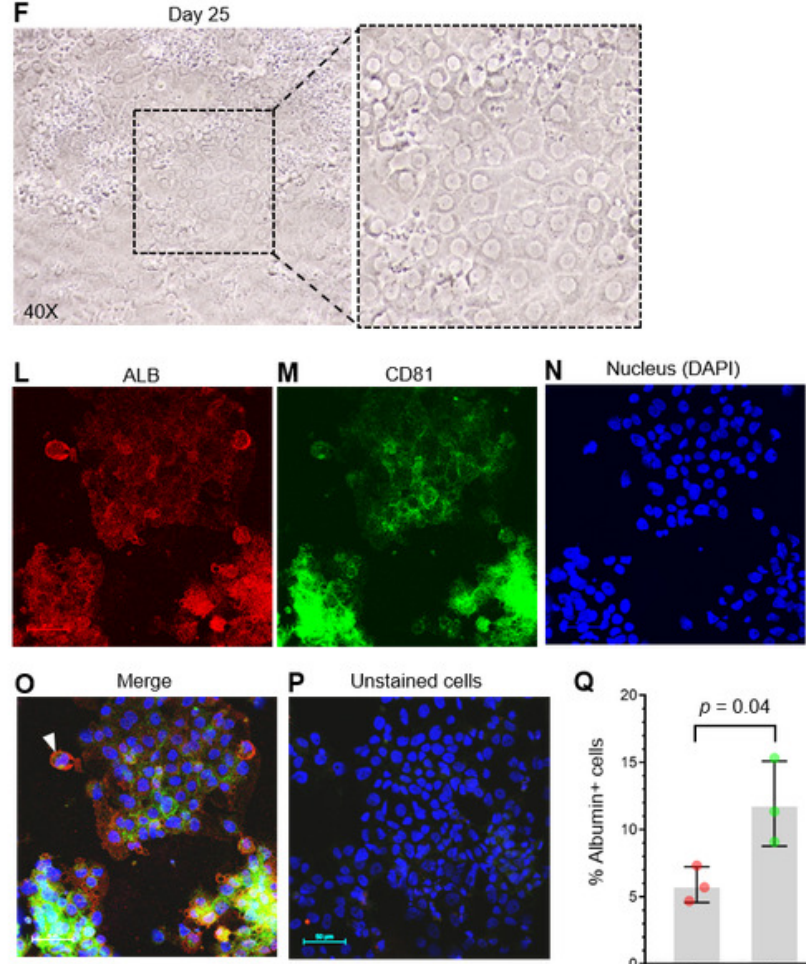

$$
\text { P Unstained cells }
$$

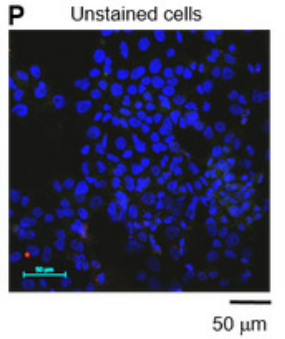

Day 15

E Day 25

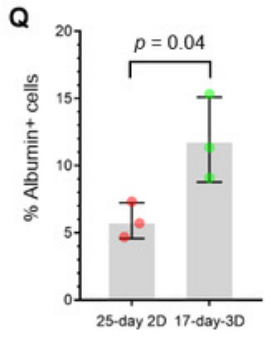

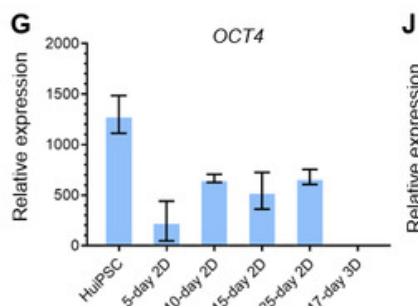
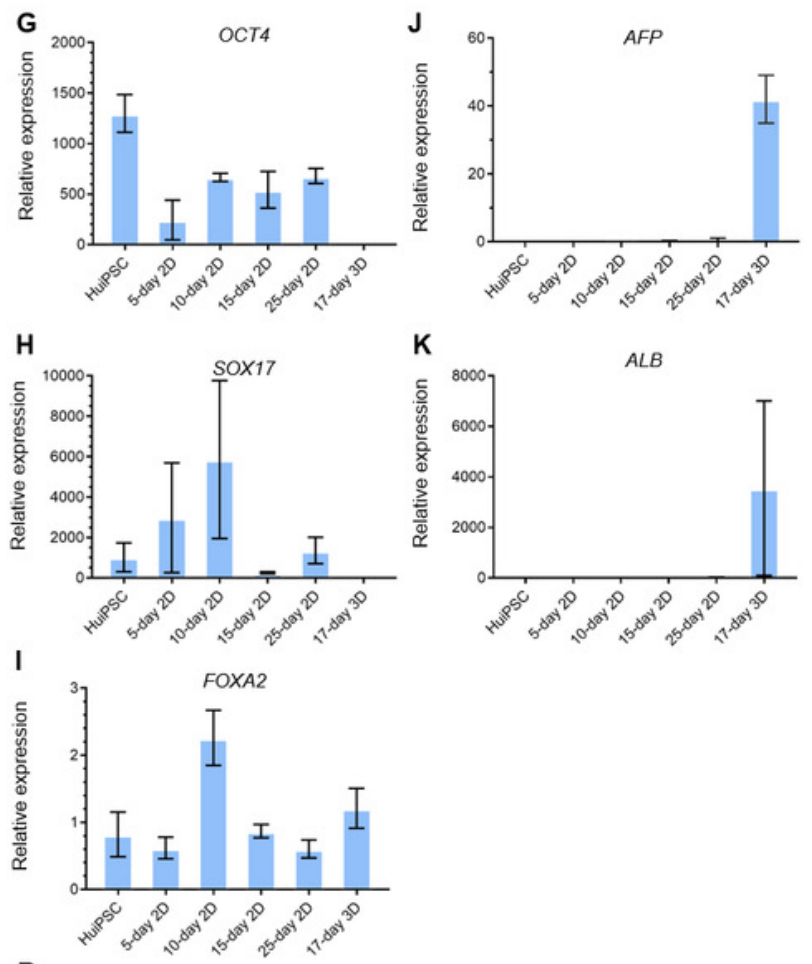

R

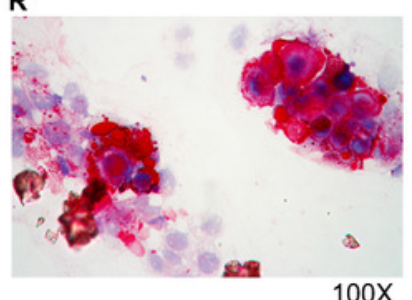




\section{Figure 2}

Organoid formation potential of the hepatic endoderm, hepatoblast, and hepatocyte phases.

(A) Schematic diagram of the methods used for assessing organoid formation ability. (B) Hepatic endoderm (day 10), hepatoblasts (day 15), and hepatocytes (day 20) were cultured in semisolid Matrigel (pink color). Liquid medium (light orange) was added on top of the Matrigel. (C-E) Ability of hepatic endoderm, hepatoblasts, and hepatocytes to form an organoid in the Matrigel-based 3D culture was assessed at day 5 of the 3D culture. Images shown were obtained with a 40X objective lens. (F-G) Two representative images of the organoid in the Matrigel-based 3D culture at day 7. (H-I) Two representative images of the organoid in the Matrigel-based 3D culture at day 10. Three independent experiments were performed and representative microscopic images were shown. 
A

\begin{tabular}{c|r} 
& $\begin{array}{c}\text { Hepatic } \\
\text { endoderm }\end{array}$ \\
\hline Endoderm &
\end{tabular}

Hepatoblast

Hepatocyte

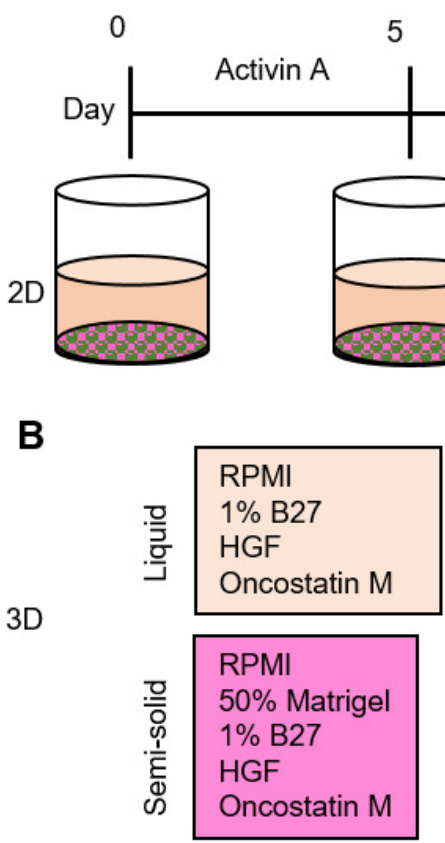

C

Day 5
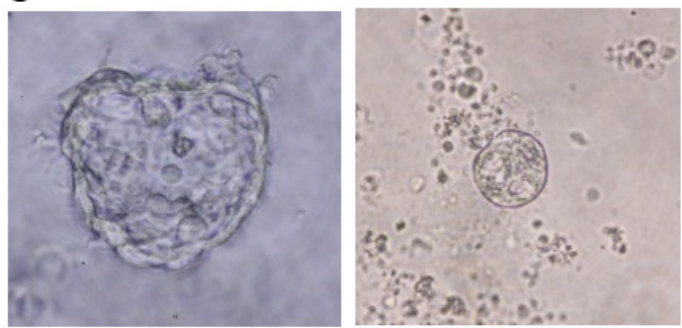

D
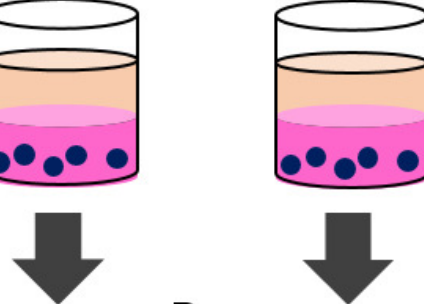

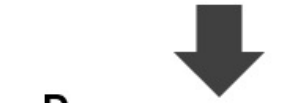

Day 7

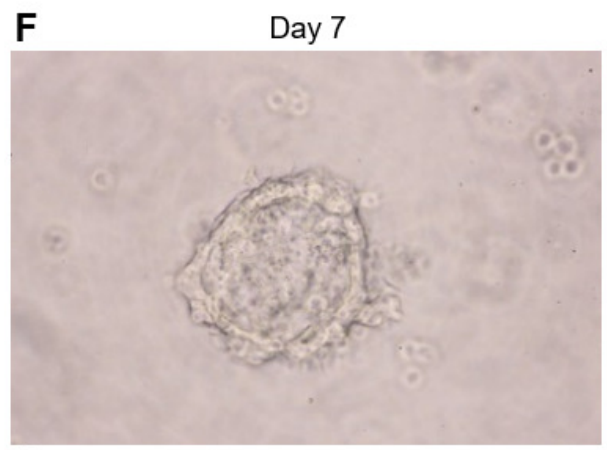

G

H

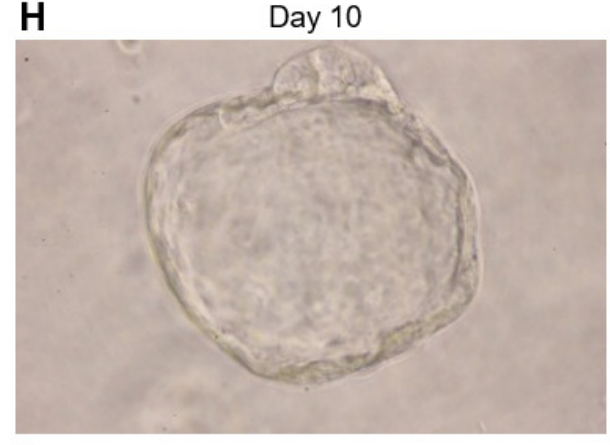

I

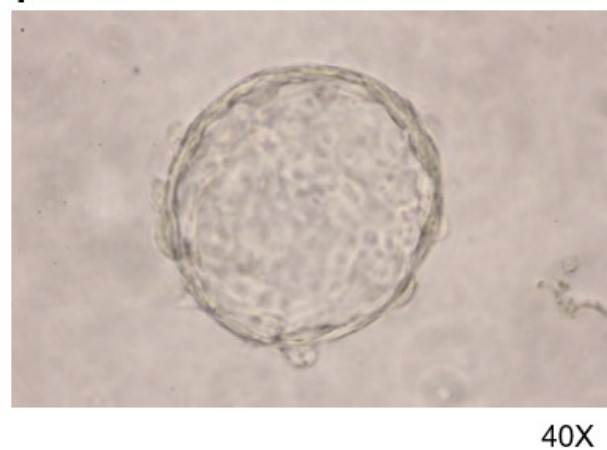




\section{Figure 3}

Morphology of hepatic endoderm-derived organoids (HEOs).

(A-C) Morphology of the HEOs at days 12,14 , and 17 postculture in Matrigel (4X objective lens). (D-F) Representative images of single HEOs are shown at high magnification (40X). (G) The outer layer of cells is shown. $(\mathrm{H})$ Zoomed-in image of the HEOs showing the cells with a polygonal shape and large nuclei (arrowheads). (I-LL) Confocal images of a day 12- and 17derived organoid showing the expression of hepatic nuclear factor 4 (HNF4), hepatocytespecific albumin (ALB) and $\alpha$-fetoprotein (AFP), cytochrome P450 3A4 (CYP4A3), and CD81, an important receptor of Plasmodium falciparum sporozoites. (MM-PP) Co-localization of CD81 and ALB in the 17-day HEO. The confocal images of organoid show the expression of CD81 (green) and hepatocyte-specific albumin (red). (QQ-TT) Zoomed-in images reveal the albumin- and CD81-expressing cells. (UU-XX) Cells stained with $2^{\text {nd }}$ antibody specific to $\lg G$ of rabbit and mouse (unstained samples) served as background control. Four independent experiments were performed and representative images were shown. (YY) Total amount of human albumin secreted within $48 \mathrm{~h}$ in the culture medium of the 25- and 40-day 2D culture and the 17- and 60-day 3D culture. Total amount (ng) of human albumin in each experiments were calculated based on number of cells. Individuals represent independent experiments. Data are the mean $\pm S D(n=3-4)$ and statistically analyzed using Student's $t$ test. Culture medium of undifferentiated HuiPSCs was used for comparison. (ZZ-EEE) Hepatocyte functions (glycogen and lipid storage, and metabolism of indocyanine) of a day 12- and 17-derived organoids. Four independent experiments were performed and representative images were shown. 


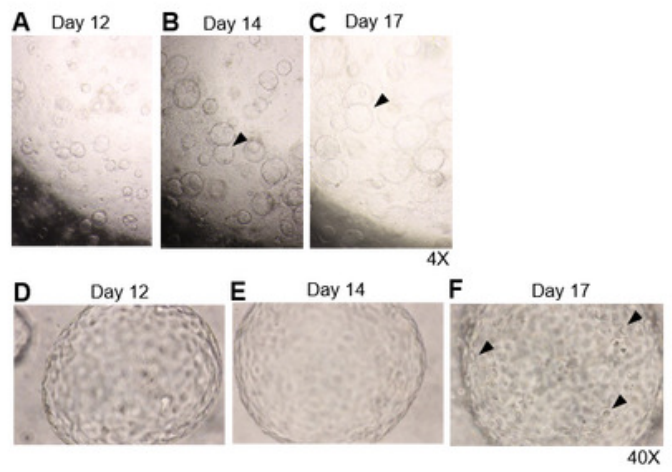

Day 12

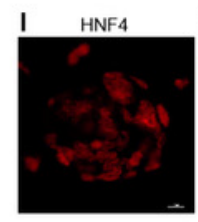

O ALB

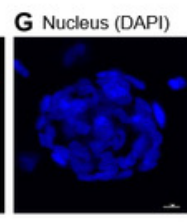

P Nucleus (DAPI)
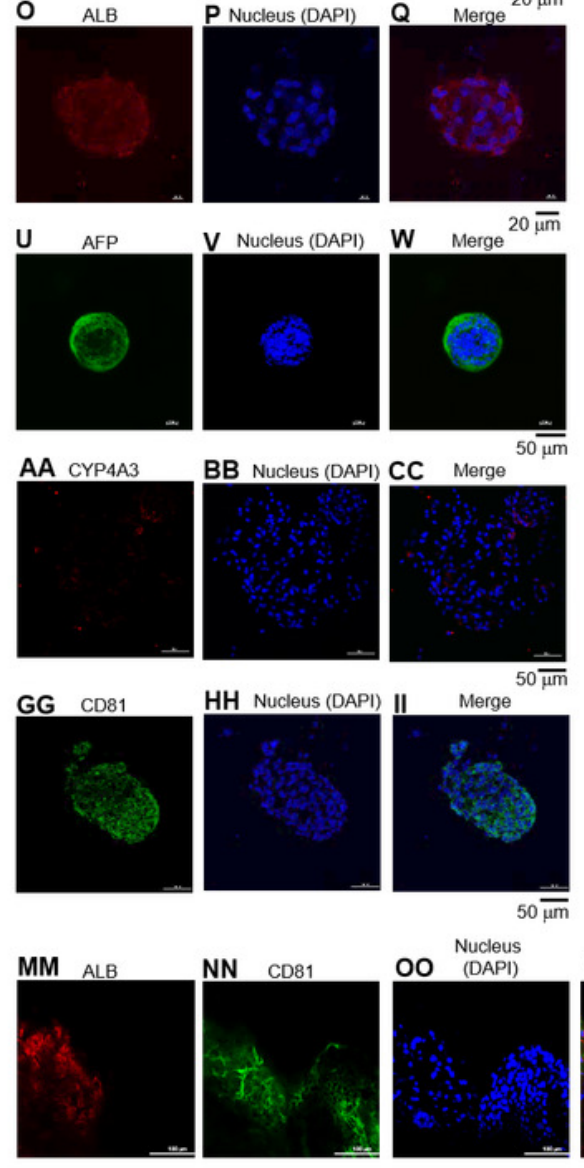

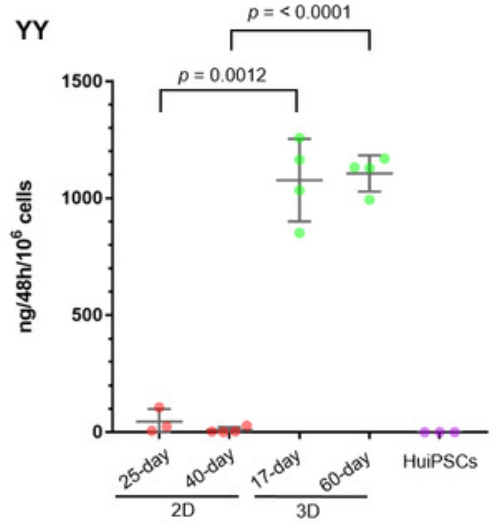

Day 17
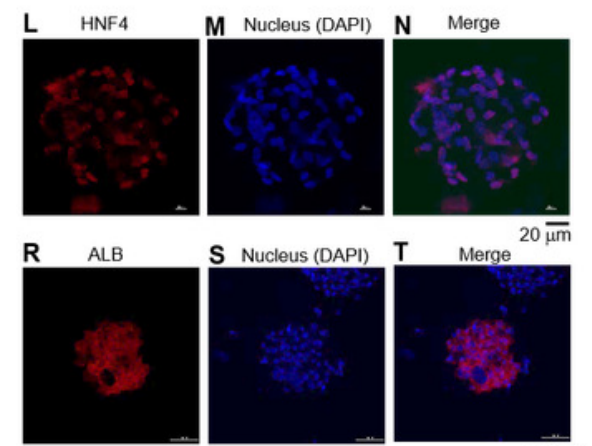

S Nucleus (DAPI)
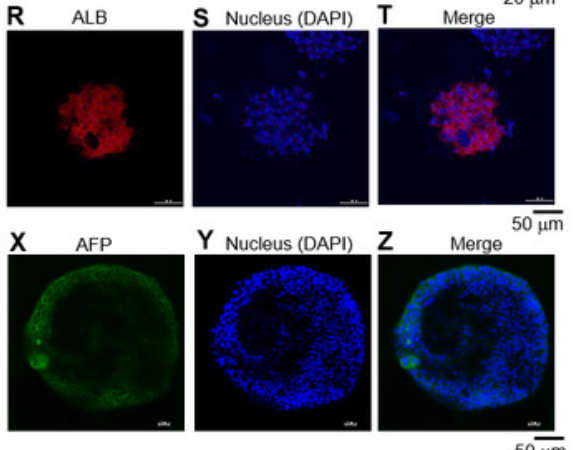

DD CYP4A3
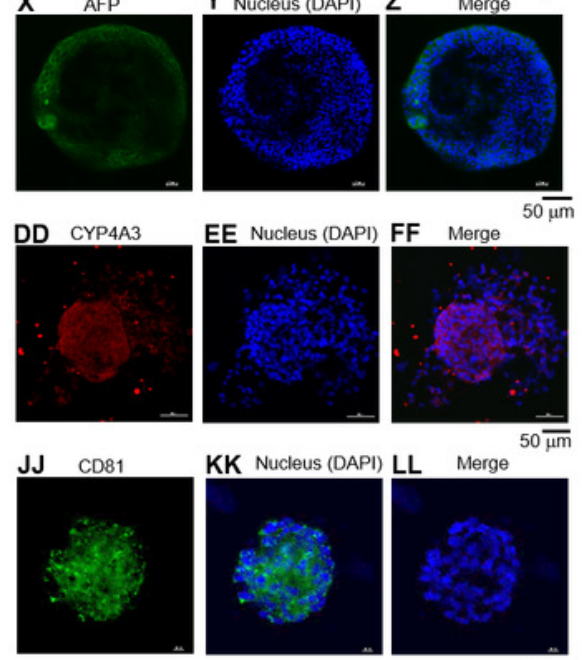

Day 12

Day 17

zz
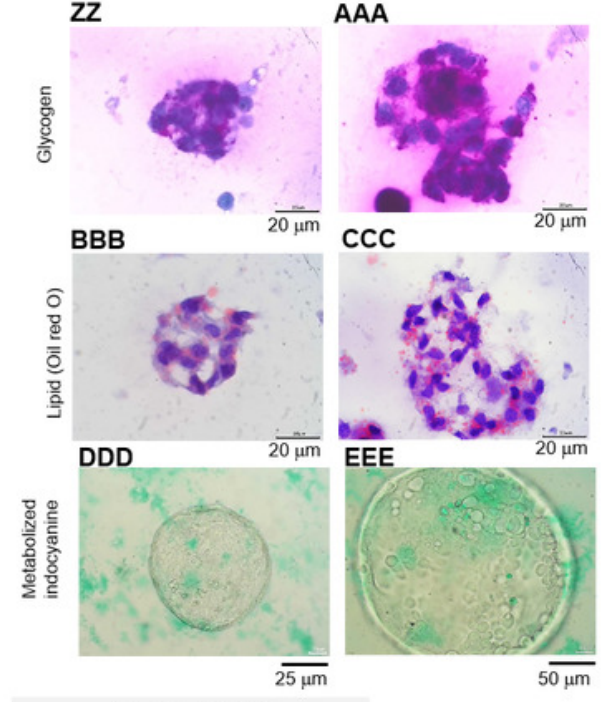

Unstained samples
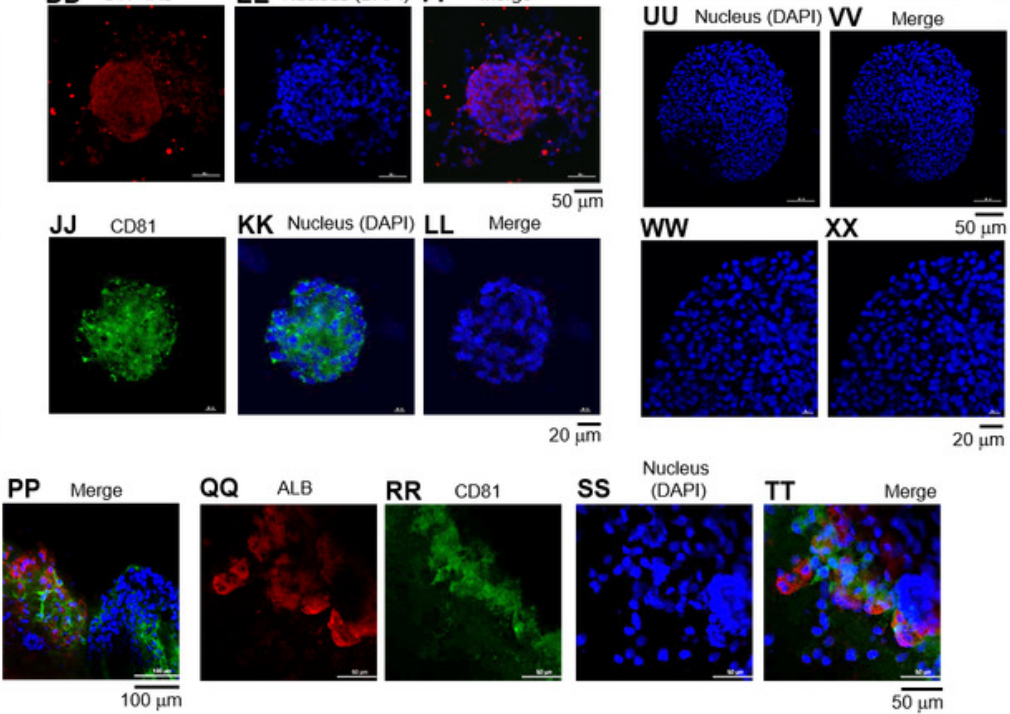


\section{Figure 4}

Minimal concentration of Matrigel needed to maintain the 3D structure of the organoid.

(A) Schematic diagram showing a method used to determine the minimum concentration of Matrigel in which the 3D structure of the organoid could be maintained. (B-E) Microscopic images of the liver organoid. The 20-day-old organoids were split to obtain $25 \%, 12.5 \%$, and $6.25 \%$ Matrigel in RPMI/B27, which were supplemented with HGF and cultured for 5 days. All images were captured using a 20X objective lens. Three independent experiments were performed and representative images were shown. 

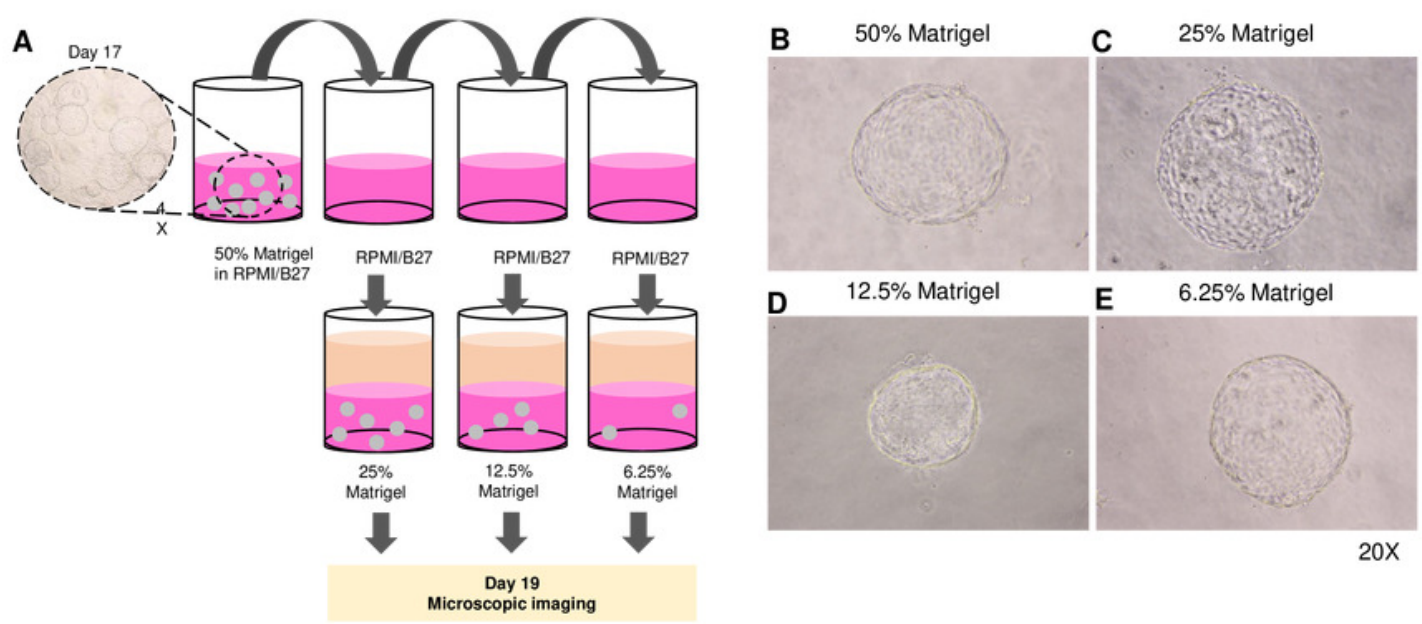


\section{Figure 5}

Long-term maintenance of the hepatic endoderm-derived organoid.

(A) Morphology of the HEOs at days 60 postculture in Matrigel. Representative images of a well of 96-well plate show the HEOs after a week of sub-culture. (B) At higher magnification, the outer layer of cells remain single. (C) Focused image of the HEOs showing the cells with a polygonal shape and large nuclei (white arrowhead) as well as binucleated cell (black arrowhead), characteristics of hepatic cells. (D-L) Confocal images of the day 60-derived HEOs showing the expression of hepatocyte-specific albumin (ALB), cytochrome P450 3A4 (CYP4A3) and CD81. (M-U) Zoomed-in confocal images shown in (D). (V-X) Hepatocyte functions (glycogen and lipid storage, and metabolism of indocyanine) of a day 60-derived organoids. Four independent experiments were performed and representative images were shown. (Y-AA) Confocal images of the day 60-derived HEOs showing the expression of EpCAM. (BB-DD) Confocal images of the day 60-derived HEOs showing the expression of CD31. 


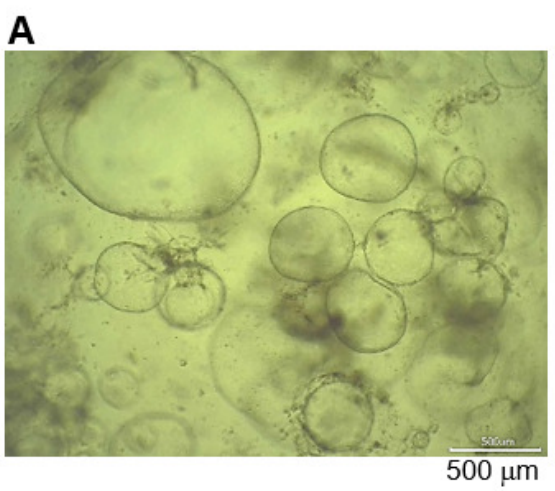

B
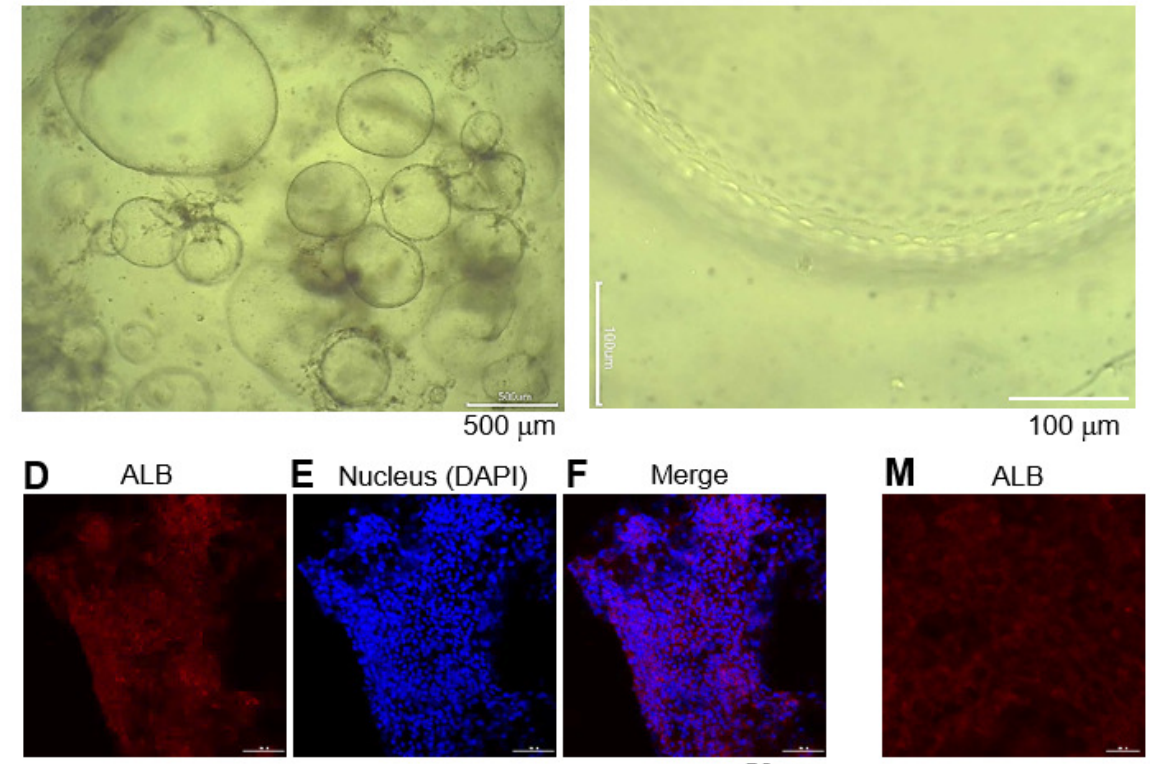

$50 \mu \mathrm{m}$

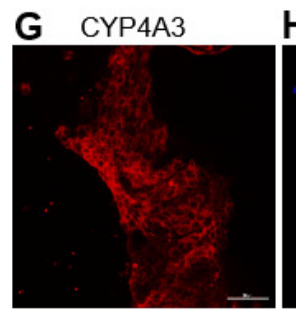

H Nucleus (DAPI)

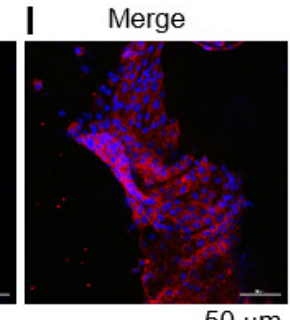

$50 \mu \mathrm{m}$
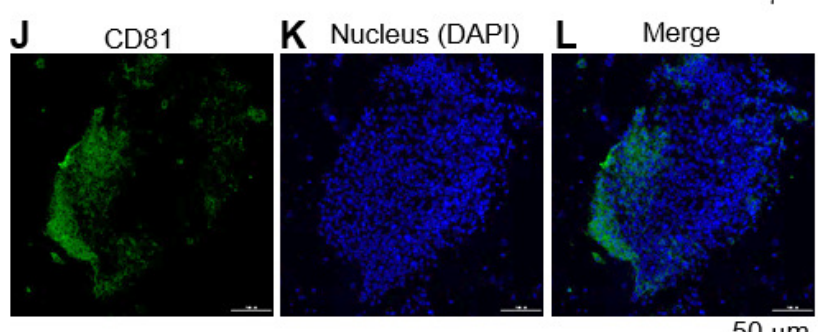

$50 \mu \mathrm{m}$

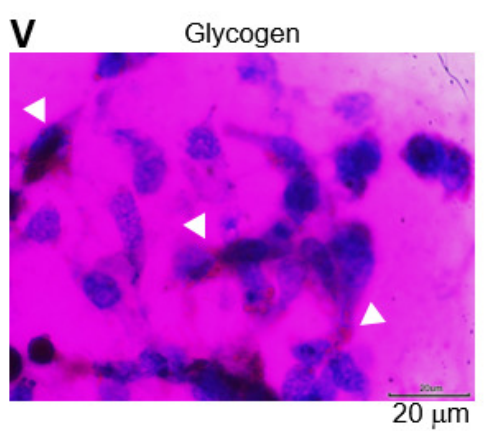

W

Lipid (Oil red O)

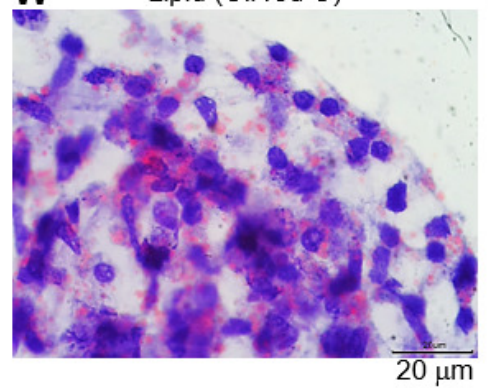

X Metabolized indocyanine
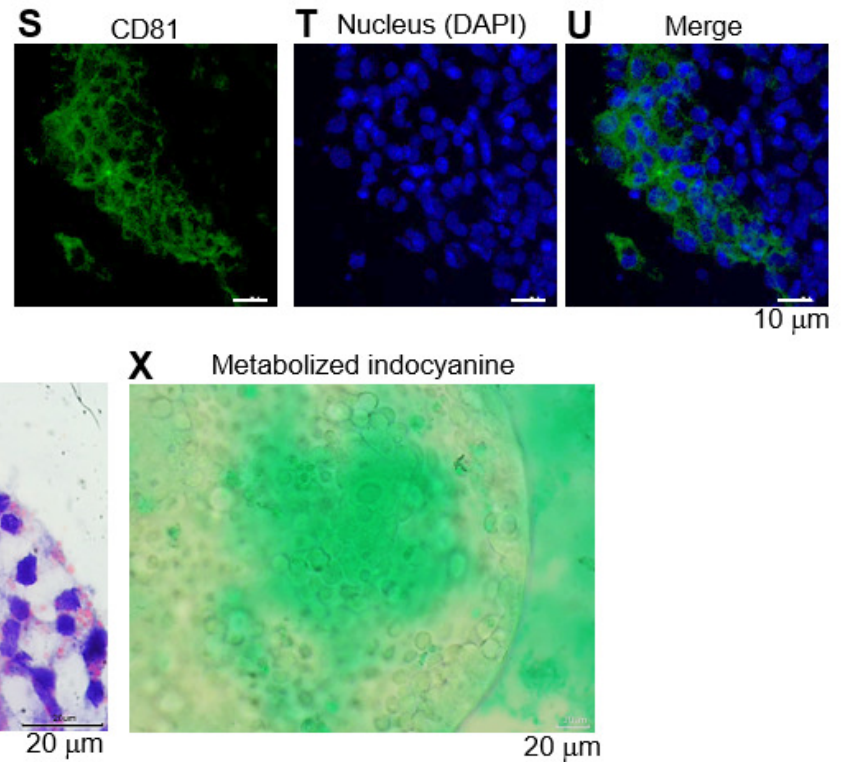

$20 \mu \mathrm{m}$ $\overline{10 \mathrm{~mm}}$
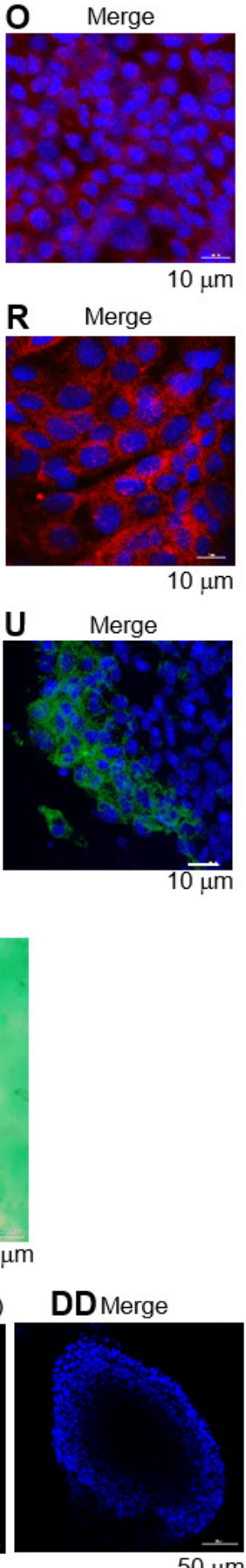

R Merge
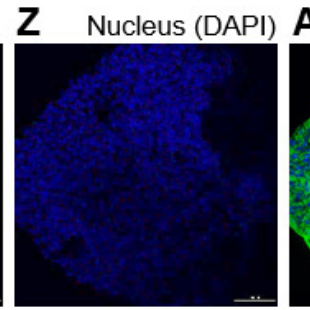
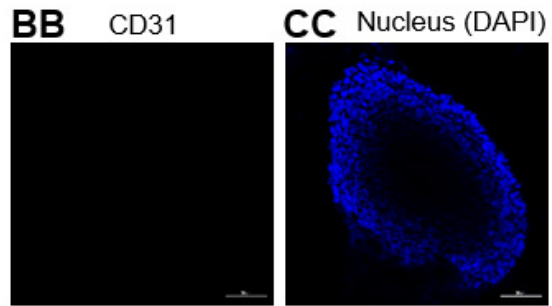


\section{Table 1 (on next page)}

Primer sequences used for quantitative PCR in the study

Primer sequences used for quantitative PCR in the study 
1 Table 1 Primer sequences used for quantitative PCR in the study

2

\begin{tabular}{|c|c|}
\hline Genes & Primers (5'-3') \\
\hline \multirow[t]{2}{*}{ OCT4 } & Fw: GCTGGAGCAAAACCCGGAGG \\
\hline & Rv: TCGGCCTGTGTATATCCCAGGGTG \\
\hline \multirow[t]{2}{*}{ SOX17 } & Fw: GAGCCAAGGGCGAGTCCCGTA \\
\hline & Rv: CCTTCCACGACTTGCCCAGCAT \\
\hline \multirow[t]{2}{*}{ FOXA2 } & Fw: TATGCTGGGAGCGGTG \\
\hline & Rv: TGTACGTGTTCATGCCGTTCA \\
\hline \multirow[t]{2}{*}{ HNF4 } & Fw: GGCAATGTGTCAGGGAGGAA \\
\hline & Rv: CAGGGATTTCAGGGGCACTT \\
\hline$A F P$ & $\begin{array}{l}\text { Fw: GCAGCCAAAGTGAAGAGG } \\
\text { Rv: TGTTGCTGCCTTTGTTTG }\end{array}$ \\
\hline$A L B$ & $\begin{array}{l}\text { Fw: AGACAAATTATGCACAGTTG } \\
\text { Rv: TTCCCTTCATCCCGAAGTTC }\end{array}$ \\
\hline
\end{tabular}

